\title{
Control del cáncer cervicouterino en Colombia: la perspectiva de los actores del sistema de salud
}

\author{
Carolina Wiesner-Ceballos, ${ }^{1}$ Raúl Hernando Murillo Moreno, ${ }^{1}$ \\ Marion Piñeros Petersen, ${ }^{1}$ Sandra Lourdes Tovar-Murillo, ${ }^{1}$ \\ Ricardo Cendales Duarte ${ }^{1}$ y Martha Cielo Gutiérrez ${ }^{1}$
}

Forma de citar Wiesner-Ceballos C, Murillo Moreno RH, Piñeros Petersen M, Tovar-Murillo SL, Cendales Duarte R, Gutiérrez MC. Control del cáncer cervicouterino en Colombia: la perspectiva de los actores del sistema de salud. Rev Panam Salud Publica. 2009;25(1):1-8.

RESUMEN Objetivo. Caracterizar, desde la perspectiva de los actores del sistema de salud, los elementos del contexto político, económico y sanitario, así como la forma en que se articulan las actividades para el control del cáncer cervicouterino en cuatro departamentos de Colombia.

Métodos. Estudio cualitativo en cuatro departamentos colombianos seleccionados por sus diferentes niveles de mortalidad por cáncer cervicouterino y de organización de las actividades de control de esta enfermedad (Boyacá, Caldas, Magdalena y Tolima). Se realizaron entrevistas semiestructuradas a funcionarios, directores de aseguradoras y de instituciones de salud públicas y privadas a niveles departamental y municipal. Se conformaron grupos focales con profesionales de las empresas aseguradoras y prestadoras de servicios. El análisis de la información se basó en la teoría fundamentada con codificaciones abiertas relacionadas con las funciones de las direcciones de salud, de las empresas aseguradoras y las acciones de los servicios de salud. Los informes técnicos se contrastaron con los testimonios.

Resultados. Se realizaron 38 entrevistas y se trabajó con 14 grupos focales $(70,9 \%$ de respuesta) y 12 informes técnicos. El cáncer cervicouterino no es percibido como una prioridad de la salud pública. El interés se centra en el flujo de dinero dentro del sistema. No se encontró una adecuada articulación entre los actores y no hay rectoría en el tema. La planificación se limita al cumplimiento de las normas. Los recursos humanos son inadecuados. No hay seguimiento de los casos positivos por la fragmentación de la población según su afiliación al sistema de salud.

Conclusiones. La orientación financiera, la planificación normativa y las dificultades de la descentralización afectan al desarrollo de las competencias, la cobertura de la población de alto riesgo y las actividades de control necesarias para el adecuado funcionamiento de los programas de tamizaje. Se requiere un programa integrado y mejor organizado en el que participen los diferentes actores del sistema de salud.

Palabras clave Neoplasias del cuello uterino, prevención y control; servicios de salud; investigación cualitativa; Colombia.

Los países desarrollados han reducido la mortalidad por cáncer cervicouterino mediante la introducción de programas

\footnotetext{
Instituto Nacional de Cancerología, Santa Fe de Bogotá, Colombia. La correspondencia se debe dirigir a Carolina Wiesner-Ceballos, Instituto Nacional de Cancerología, Calle 1 No. 9-85, Santa Fe de Bogotá, Colombia. Correo electrónico: cwiesner@ cancer.gov.co
}

de tamizaje $(1,2)$. Sin embargo, en los países de ingresos medios y bajos no se ha observado una reducción similar y se estima que $80,6 \%$ de los casos y $83,1 \%$ de las muertes por esta enfermedad en el año 2000 ocurrieron en países en vías de desarrollo (3). En América Latina, a pesar de la existencia de programas de tamizaje mediante pruebas citológicas, la mortalidad por cáncer cervicouterino no ha disminuido y en la mayoría de los países ha aumentado (4). Esto se atribuye a factores relacionados con problemas de sensibilidad de las pruebas empleadas, la insuficiente organización y la baja calidad de los programas existentes (5). Aunque se introduzcan pruebas más sensibles, como las basadas en el ADN 
del virus del papiloma humano, el análisis de los problemas que han incidido en esta situación resulta pertinente, ya que independientemente de las tecnologías utilizadas, algunos factores relacionados con los programas, como la cobertura, la calidad y el seguimiento, serán siempre determinantes para la detección temprana de esta enfermedad.

Colombia implementó en 1990, antes de la reforma de su sistema de salud, un programa para el control del cáncer cervicouterino, pero al igual que en otros países latinoamericanos, no se ha logrado reducir significativamente la mortalidad por esta causa y la tasa ajustada en 2002 fue de 18,2 por 100000 mujeres (6).

El Sistema General de Seguridad Social en Salud (SGSSS) colombiano se basa en la competencia regulada de servicios públicos y privados dentro de un modelo de aseguramiento descentralizado y modulado por el Estado (7-9). En ese contexto, las entidades públicas promueven la realización de pruebas citológicas del cuello uterino, mientras la toma del examen, el diagnóstico y el tratamiento de la enfermedad se hacen principalmente a través de empresas aseguradoras, la mayoría de ellas privadas (10). Las mujeres que en el año 2004 no tenían seguro de salud $(32,9 \%)$ (11) se realizaban la prueba citológica en dependencias de los servicios municipales de salud y recibían la complementación diagnóstica y el tratamiento en dependencias de los servicios departamentales. $^{2}$

Las acciones desarrolladas en Colombia para el control de esta enfermedad son similares a las emprendidas en otros países de América Latina ya que, a pesar de las diferencias en los sistemas de salud, el contexto socioeconómico es similar. El objetivo del presente artículo es caracterizar, desde la perspectiva de los actores del sistema, los elementos del contexto político, económico y sanitario, y la forma en que se articulan las actividades de tamizaje del cáncer cervicouterino en el trabajo cotidiano de los servicios de salud en cuatro departamentos de Colombia.

\section{MATERIALES Y MÉTODOS}

Se realizó un estudio cualitativo en el que se seleccionaron por conveniencia cuatro escenarios según el nivel de organización de las actividades programadas

\footnotetext{
2 En Colombia, los departamentos equivalen a los estados o las provincias en otros países latinoamericanos.
}

CUADRO 1. Fuentes de información en los cuatro departamentos seleccionados. Colombia, 2004-2006

\begin{tabular}{|c|c|c|c|c|c|}
\hline Entrevistas & No. & Grupos focales & No. & Informes técnicos ${ }^{a}$ & No. \\
\hline Directores de salud pública & 4 & Secretarías de salud & 2 & Plan de atención básica 2004 & 4 \\
\hline Directores de aseguramiento & 4 & Aseguradores & 4 & Informe de gestión, 2004 & 4 \\
\hline Directores de epidemiología & 4 & $\begin{array}{l}\text { Funcionarios de centros } \\
\text { de tamizaje }\end{array}$ & 2 & Boletín epidemiológico & 4 \\
\hline $\begin{array}{l}\text { Directores de prestación } \\
\text { de servicios }\end{array}$ & 4 & $\begin{array}{l}\text { Centros de patología } \\
\text { cervicouterina }\end{array}$ & 2 & & \\
\hline $\begin{array}{l}\text { Directores de laboratorios } \\
\text { de salud pública }\end{array}$ & 4 & Laboratorios de citología & 2 & & \\
\hline $\begin{array}{l}\text { Coordinadores de salud sexual } \\
\text { y reproductiva }\end{array}$ & 4 & $\begin{array}{l}\text { Instituciones de tratamiento } \\
\text { de cáncer }\end{array}$ & 2 & & \\
\hline $\begin{array}{l}\text { Coordinadores de cáncer } \\
\text { cervicouterino }\end{array}$ & 4 & & & & \\
\hline $\begin{array}{l}\text { Secretarías de salud de las } \\
\text { capitales de departamento }\end{array}$ & 4 & & & & \\
\hline $\begin{array}{l}\text { Coordinadores del Plan de } \\
\text { Atención Básica }\end{array}$ & 4 & & & & \\
\hline $\begin{array}{l}\text { Director del registro poblacional } \\
\text { de cáncer }\end{array}$ & 2 & & & & \\
\hline Total & 38 & & 14 & & 12 \\
\hline
\end{tabular}

a Los informes técnicos revisados fueron los siguientes: 1) Dirección Territorial de Salud de Caldas. Indicadores básicos en salud. Manizales: Gobernación de Caldas; 2004. 2) Secretaría de Salud del Tolima. Indicadores básicos en salud del Tolima. Ibagué: Gobernación del Tolima; 2004. 3) Instituto Seccional de Salud de Boyacá. Diagnóstico de la situación de salud de Boyacá. Tunja: Gobernación de Boyacá; 2004. 4) Secretaría de Desarrollo de la Salud del Magdalena. Tablas de estadísticas vitales 2004-2005. Santa Marta: Gobernación del Magdalena; 2005. 5) Dirección Territorial de Salud de Caldas. Plan de atención básica: 2004-2007. Manizales: Gobernación de Caldas; 2004. 6) Dirección Territorial de Salud de Caldas Informe de Gestión: 2004-2007. Manizales: 2004; 7) Secretaría de Salud del Tolima. Plan de atención básica. Ibagué: Gobernación del Tolima; 2004; 8) Secretaría de Salud del Tolima. Informe de gestión. Ibagué: Gobernación del Tolima; 2004; 9) Instituto Seccional de Salud de Boyacá. Plan de atención básica 2004. Tunja: Gobernación de Boyacá; 2004; 10) Instituto Seccional de Salud de Boyacá. Informe de gestión 2004. Tunja: Gobernación de Boyacá; 2004; 11) Secretaría de Desarrollo de la Salud del Magdalena. Plan de atención básica 2004-2005. Santa Marta: Gobernación del Magdalena; 2005. 12) Secretaría de Desarrollo de la Salud del Magdalena. Informe de gestión 2004-2005. Santa Marta: Gobernación del Magdalena; 2005.

y la mortalidad por cáncer cervicouterino: dos departamentos tenían un adecuado desempeño en el número de pruebas citológicas realizadas, pero con tasas variables de mortalidad (Boyacá y Caldas) y dos departamentos tenían un inadecuado desempeño en la toma de muestras para las pruebas citológicas e igualmente, tasas variables de mortalidad (Magdalena y Tolima) (12).

Las preguntas centrales de la investigación fueron: ¿cuál es la percepción acerca de la situación de la salud sexual y reproductiva y del cáncer cervicouterino en su departamento?, ¿cuáles áreas de las direcciones departamentales de salud participan en la planificación, gestión y evaluación de las actividades de detección temprana del cáncer cervicouterino?, ¿cómo se articulan los diferentes actores del SGSSS para cumplir con los objetivos de prevención y detección temprana del cáncer cervicouterino?

Se realizaron entrevistas semiestructuradas, se conformaron grupos focales y se revisaron y analizaron informes técnicos relacionados con el tema (cuadro 1). Se entrevistaron funcionarios con responsabilidades en el control del cáncer cervicouterino de los cuatro departamentos (tanto a nivel departamental como municipal), directores de las empresas aseguradoras y directores de las instituciones (públicas y privadas) prestadoras de servicios de salud. Los grupos focales se conformaron con profesionales de las empresas aseguradoras y prestadoras de servicios (enfermeros, ginecólogos, directores de instituciones para el tratamiento del cáncer, técnicos en citología y patólogos). Tanto en las entrevistas como en los grupos focales se alcanzó la saturación teórica.

La información se recolectó entre agosto de 2005 y febrero de 2006. Las entrevistas se registraron en cintas magnéticas en condiciones que garantizaran la confidencialidad del informante. Para el análisis se utilizó el modelo propuesto por Glasser y Strauss para el desarrollo de la teoría fundamentada $(13,14)$. Con apoyo del programa computacional ATLAS $/ \mathrm{ti}^{\circledR}$ se realizó inicialmente una codificación abierta en dos temas centrales: las funciones que deben cumplir las direcciones de salud y las acciones de los programas de control del cáncer cervicouterino, es decir, la promoción y la re- 
alización de las pruebas citológicas de tamizaje, el tratamiento, el control de la calidad, el seguimiento de los casos y la evaluación del programa. Se elaboraron notas registradas como ideas, reflexiones e hipótesis. Los informes técnicos se contrastaron con los testimonios de los entrevistados. Los resultados se validaron en talleres de discusión con funcionarios de las cuatro secretarías departamentales de salud que participaron en el estudio.

Este trabajo recibió la aprobación del Comité de Ética del Instituto Nacional de Cancerología de Colombia y es parte de la iniciativa aprobada para evaluar las actividades de detección temprana del cáncer cervicouterino en Colombia.

\section{RESULTADOS}

Se realizaron 38 entrevistas y se trabajó con 14 grupos focales, con 70,9\% de respuestas (105 de 148 invitados); también se revisaron 12 informes técnicos de los cuatro departamentos (cuadro 1). Se encontraron algunas diferencias entre los departamentos que registran mortalidades altas y los que registran mortalidades bajas, particularmente en la organización de las acciones de detección temprana.

\section{Percepción de la magnitud del problema del cáncer cervicouterino}

De acuerdo con los testimonios y los informes técnicos revisados, en ninguno de los departamentos se percibía al cáncer cervicouterino como una prioridad y solo en uno de ellos se contaba con información sobre los problemas generados por los tumores malignos, pero sin la descripción específica de la situación del cáncer cervicouterino. De acuerdo con estas fuentes de información, la infección por el VIH/sida y los problemas reproductivos son prioridades mayores, lo cual define una asignación baja de recursos para atender el cáncer cervicouterino (cuadro 2: AL1; cuadro 3: P1).

Una de las razones aducidas de la poca importancia dada al cáncer cervicouterino es el llamado "silencio epidemiológico", que se manifiesta en la falta de datos y la ausencia de sistemas de información. El escaso interés en este tema se limita a la auditoria del cumplimiento de los contratos (metas en la toma de muestras para las pruebas citológicas), lo que se relaciona con el flujo de dinero dentro del sistema (cuadro 2: AL2).

CUADRO 2. Testimonios escogidos de las autoridades locales entrevistadas

\begin{tabular}{lll}
\hline Código & No. de la \\
\hline entrevista
\end{tabular}

AL1 “. . al VIH, que se le dio más prioridad debido a que es un objetivo a nivel nacional y a nivel departamental."

AL2 "Esta niña que maneja lo de salud sexual y reproductiva, ella tiene a cargo interventorías de varios contratos, entonces ella mira cómo van, si se está cumpliendo el objeto del contrato."

AL3 "El recurso humano ha sido cambiante, rotatorio. . . de pronto había personas que no manejaban el perfil de lo que significa este componente."

AL4 "Cada persona llega y quiere trabajarlo como mejor le parece y con otras directrices. . ."

AL5 "Entonces yo hice un gol al PAB a . . yo metí también el apoyo a la lecturab como un apoyo para las vinculadas ${ }^{c}$. . . Dejé un proyecto donde apoyaba en concurrencia a los municipios y en 2004. . . un apoyo para la lectura, porque es que tomaban las citologías ${ }^{d}$ y las instituciones con el problema no mandaban a leer eso."

AL6 "Nosotros hacemos más actividades de promoción, ellos hacen la toma de citología, la lectura; pero ellos trabajan totalmente aparte y rinden unos informes acá de acuerdo a unas matrices de programación. . . pero no trabajamos articuladamente. . ."

AL7 "En este año pensamos reunirnos con las aseguradoras, los alcaldes y los gerentes de las instituciones prestadoras de servicios, es decir, con cada uno de los integrantes del sector salud para firmar algunos acuerdos para que se garantice el cumplimiento de la programación de las actividades de promoción y prevención."

AL8 "Todos sabíamos que eso era obligatorio. . . nos llamaba la atención que había personas que están muriendo de cáncer cervicouterino, pero nosotros no las veíamos. Entonces dijimos: ¿qué es lo que está pasando? Vamos a empezar a revisar las placas de las niñas que están leyendo en los municipios. Pero solamente tenemos dos municipios que cumplen con esa exigencia, el resto no se ha podido porque son innumerables las niñas que están leyendo solas sin controles internos siquiera."

a PAB: Plan de Atención Básica, componente de salud colectiva dentro de la Ley 100 de seguridad social en salud.

b Leer: se refiere al análisis de las pruebas citológicas.

c Vinculada: término acuñado entre los actores del sistema para denominar a la población que no tiene cobertura de ningún sistema de seguro por no tener capacidad de pago ni subsidio del estado.

d Citología: prueba citológica.

\section{CUADRO 3. Testimonios seleccionados de los prestadores según el grupo focal}

\begin{tabular}{|c|c|c|}
\hline Código & Testimonio & $\begin{array}{l}\text { No. del } \\
\text { grupo focal }\end{array}$ \\
\hline P1 & $\begin{array}{l}\text { "Los hospitales reciben recursos para lo de las citologías, }{ }^{a} \text { pero no le dan importancia } \\
\text { a ese programa." }\end{array}$ & 17 \\
\hline $\mathrm{P} 2$ & "No es fácil el seguimiento si no se tiene una base de datos." & 16 \\
\hline P3 & $\begin{array}{l}\text { ". . cuando el Ministerio dice hagamos esto, hablamos el mismo idioma. . . en este } \\
\text { momento no tenemos un lineamiento claro." }\end{array}$ & 23 \\
\hline P4 & $\begin{array}{l}\text { "Es triste, ¿por qué tienen que manejar los recursos allá si nosotros tenemos la población } \\
\text { aquí? Nosotros tenemos la oportunidad de aprovechar esos recursos y detectar } \\
\text { oportunamente tanta problemática, pero por la ley y por la norma no lo podemos hacer." }\end{array}$ & 18 \\
\hline P5 & ". . para que tomen la muestra hay accesibilidad, pero para el procesamiento no lo hay. .." & ." 20 \\
\hline P6 & $\begin{array}{l}\text { "Cuando duramos mucho tiempo sin el patólogo nos toca buscar ayuda donde los amigos } \\
\text { patólogos para que nos revisen las placas." }\end{array}$ & 27 \\
\hline P7 & $\begin{array}{l}\text { "Ellos mandan citologías por allá de hace tiempo. A las pacientes no las controlan. Esas } \\
\text { son las pacientes que a cada rato ve uno que NIC } 1 \text {, NIC 2, y NIC 3; y al año ipum! } \\
\text { cáncer. .." }\end{array}$ & 17 \\
\hline P8 & $\begin{array}{l}\text { "En cáncer cervicouterino? Para ellos no es importante el laboratorio. Ellos nos dicen: } \\
\text { pues si se acabó el alcohol jno lea!c No le dan la importancia que esto tiene, como se } \\
\text { venía haciendo cuando el programa estaba fortalecido. En este momento no tenemos ni } \\
\text { mamá ni papá..." }\end{array}$ & 23 \\
\hline
\end{tabular}

a Citologías: pruebas citológicas.

b NIC: neoplasia intraepitelial celular.

c Leer: se refiere al análisis de las pruebas citológicas.

La población está fragmentada según el tipo de afiliación al seguro y la información que genera cada empresa aseguradora no aporta la información necesa- ria para tomar decisiones relacionadas con el seguimiento de las mujeres con lesiones cervicouterinas. La información se obtiene de los registros de procedi- 
CUADRO 4. Testimonios escogidos de los aseguradores según el grupo focal

\begin{tabular}{|c|c|c|}
\hline Código & Testimonio & $\begin{array}{l}\text { No. del } \\
\text { grupo focal }\end{array}$ \\
\hline AS2 & “. . los registros médicos no permiten tomar decisiones oportunas de lo que está & \\
\hline & sucediendo específicamente en esta patología." & 2 \\
\hline AS3 & $\begin{array}{l}\text { ". . falta articulación, no hay verdaderamente un lineamiento vertical y se ha deteriorado } \\
\text { logística, administrativa y eficientemente el programa." }\end{array}$ & 25 \\
\hline AS4 & $\begin{array}{l}\text { ". . yo no sé si el cáncer de cuello uterino necesitaría algo tan especial como cuando } \\
\text { se dice jornada de vacunación." }\end{array}$ & 23 \\
\hline AS5 & $\begin{array}{l}\text { "Se hicieron unas capacitaciones por parte de la Secretaría Departamental. . y toda una } \\
\text { serie de capacitaciones que se han hecho en promoción y prevención, que por lo menos } \\
\text { nosotros nunca. . . nunca he estado involucrada en este tipo de capacitaciones." }\end{array}$ & $\begin{array}{l}a \\
S\end{array}$ \\
\hline AS6 & $\begin{array}{l}\text { "Las plantillas de programación de prevención de las mujeres de las EPS a están todas } \\
\text { centralizadas con el Ministerio de Salud y resulta que lo que nos llega acá es diciendo: } \\
\text { mire, esta es su programación, listo, sus metas de cumplimiento." }\end{array}$ & 15 \\
\hline AS7 & $\begin{array}{l}\text { ". . uno ve que casi siempre son las mismas usuarias. . estamos quedándonos con las } \\
\text { que llegan siempre. . . a los tres meses repite la usuaria; el esquema } 1-1-3^{b} \text { no se está } \\
\text { ejecutando tal cual y, lo que hablaban hace un momento, estamos trabajando en función } \\
\text { de la cantidad." }\end{array}$ & 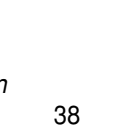 \\
\hline AS9 & $\begin{array}{l}\text { ". .el Día de la Mujer, entonces todo citologías }{ }^{\circ} \text { y terminó marzo y no supimos cuáles } \\
\text { fueron los resultados." }\end{array}$ & 16 \\
\hline AS9 & $\begin{array}{l}\text { "Cuando salió la norma, inmediatamente había días en que nos llegaban } 10 \text { y } 12 \\
\text { autorizaciones para colposcopia de pacientes que ya llevaban seis meses o un año." }\end{array}$ & 1 \\
\hline AS10 & $\begin{array}{l}\text { ". . para allá y para acá, el usuario va para un lado. . . mejor dicho es que se le ve que } \\
\text { tiene la cara del diagnóstico. . . el vigilante lo ve en la puerta y dice esta señora tiene } \\
\text { cáncer, ¿sí? Y sin embargo como no tiene la biopsia, en ese caso no lo podemos recibir, } \\
\text { o sea es ley." }\end{array}$ & $r$ \\
\hline
\end{tabular}

a EPS: Empresa Promotora de Salud, denominación genérica dada a las empresas aseguradoras en salud que atienden a la población trabajadora o con capacidad de pago y sus beneficiarios.

b El llamado esquema 1-1-3 consistente en realizar dos pruebas citológicas anuales y en caso de ser estas negativas continuar con una citología cada tres años.

c Citologías: pruebas citológicas.

mientos médicos diseñados para la facturación, que no sirve para la toma de decisiones (cuadro 3: P2; cuadro 4: AS2).

\section{Organización del programa}

Dirección y rectoría. Las estructuras de las direcciones de salud de los cuatro departamentos son similares. Las áreas de aseguramiento, salud pública y desarrollo (prestación) de servicios comparten responsabilidades en el control del cáncer. El grupo de aseguramiento vigila la afiliación al sistema de salud de la población de bajos recursos económicos que cuenta con subsidios por parte del Estado; el grupo de salud pública promueve la realización de las pruebas citológicas; y el área de desarrollo de servicios debe gestionar la prestación de servicios oportunos, eficientes y de alta calidad a la población subsidiada. Según lo expresado por los funcionarios entrevistados, después de la reforma del sistema nacional de salud de 1993 no ha habido una adecuada articulación en estas funciones y se percibe la falta de una entidad rectora que cuente con los recursos legales y administrativos para llevar a cabo la dirección, la asistencia técnica y la evaluación de las actividades relacionadas con el tema (cuadro 3: P3; cuadro 4: AS3, AS4).

Esta situación se refleja en la planificación de las acciones, donde se observa claramente que el interés se limita a cumplir con las funciones especificadas en la norma vigente, según lo expresaron los profesionales de instituciones privadas (cuadro 4: AS5). Adicionalmente, la concentración de responsabilidades (por recortes en la nómina), la alta rotación de personal y la baja continuidad en la gestión y el desarrollo de los planes han debilitado la capacidad técnica (cuadro 2: AL3, AL4). Los departamentos en los que los recursos humanos tienen una mayor permanencia proponen acciones que reflejan un mayor compromiso con las tareas de detección temprana, pero en esos casos se tiene la percepción de estar infringiendo las normas (cuadro 2: AL5). En consecuencia, más que una adecuada planificación, lo realizado por las direcciones de salud en los departamentos y los municipios se ve como el cumplimiento de las actividades y las funciones establecidas (cuadro 2: AL6).

En ninguno de los departamentos se encontró información detallada sobre la población a la que se deben dirigir las acciones de detección temprana del cáncer cervicouterino (mujeres de 25 a 69 años); los datos existentes se circunscriben a lo relativo al flujo de recursos, como la facturación de actividades para la población de bajos recursos asegurada o información sobre la población sin seguro de salud a la que también se debe atender (cuadro 4: AS6). El interés de las instancias de dirección y rectoría también se centraron en temas de presupuestos y de flujo de recursos, más que en la planificación de intervenciones.

Esta situación dificulta la interacción y la articulación de los niveles departamental y municipal, y hace que ambas instancias encuentren limitantes para el adecuado cumplimiento de los objetivos establecidos para el control del cáncer, no solo por las dificultades en la planificación sino además por no contar con los recursos necesarios (cuadro 3: P4). En la actualidad, las direcciones de salud tienen conciencia de la situación y ven la necesidad de establecer acuerdos entre los diferentes actores del sistema, pero siempre orientados al cumplimiento de la norma y las metas definidas en ella (cuadro 2: AL7). De hecho, se identificó la búsqueda de alternativas programáticas, como el caso de uno de los departamentos en el que se delegó la responsabilidad del control del cáncer a una universidad pública que propuso un programa descentralizado. Sin embargo, esta iniciativa se vio limitada por las normas del nuevo sistema de salud y se limitó al cumplimiento de lo establecido $\mathrm{y}$, por tanto, a la franja de población para la que se le asignaron recursos.

Atención a las pacientes. En aras de cumplir con las metas de cobertura de las pruebas citológicas, las entidades territoriales de salud y las aseguradoras realizan las pruebas a adolescentes o las repiten antes del año, lo que hace que no se cumpla el llamado esquema 1-1-3, consistente en realizar dos citologías anuales y en caso de ser estas negativas continuar con una citología cada tres años (cuadro 4: AS7). Las direcciones de salud no cuentan con información sobre el acceso y la cobertura de las pruebas citológicas en la población cubierta por las empresas aseguradoras, excepto en lo relativo a las metas de programación descritas. Esta información no les permite hacer el seguimiento adecuado de las mujeres. En este sentido, en la dirección 
de salud de uno de los departamentos estudiados que presentaba una alta mortalidad y un adecuado cumplimiento en el número de pruebas citológicas realizadas, se capacitaron médicos generales en colposcopia como una alternativa para mejorar la oportunidad de atención; sin embargo, surgieron dificultades, como la negativa de las empresas aseguradoras a pagar las colposcopias debido a que, según la norma, estas deben estar realizadas por ginecólogos.

El análisis de las muestras se retrasa con frecuencia debido a dificultades para su transportación o la falta de continuidad de los contratos establecidos entre las empresas aseguradoras y las entidades territoriales de salud con los laboratorios. De hecho, en algunas ocasiones se realizan campañas para la toma de muestras para las pruebas citológicas, pero luego no se conocen los resultados, lo que igualmente refleja la prioridad dada a las metas de programación y no a los objetivos (cuadro 3: P5, P6; cuadro 4: AS8).

Hasta diciembre de 2004, el plan de servicios vigente para las mujeres aseguradas de escasos recursos económicos no incluía la colposcopia, lo que representaba un obstáculo importante para el acceso al diagnóstico. La posterior inclusión de la colposcopia generó una alta demanda por las necesidades acumuladas y esto, a su vez, generó un incremento en el número de casos incidentes (cuadro 4: AS9). De manera similar, los trámites que deben realizar las mujeres ante su asegurador para obtener la autorización de los procedimientos médicos imponen dificultades para la atención de pacientes con diagnóstico de cáncer invasor, particularmente si este se basa en criterios clínicos. Esta situación es especialmente crítica en la población asegurada de bajos recursos económicos ya que, como se mencionó anteriormente, el procedimiento diagnóstico no está incluido en el plan de servicios (cuadro 3: P7; cuadro 4: AS10).

Control de la calidad. Solo en uno de los departamentos, que contaba con baja mortalidad e inadecuado cumplimiento de las metas de cobertura de citologías, el laboratorio de salud pública realizaba controles de calidad externos y cumplía su doble función de analizar las pruebas citológicas y hacer el control externo de otros laboratorios. Los problemas en la calidad de las pruebas son conocidos, pero aun así, el único laboratorio que cumplió con ambas funciones no tenía la cobertura y la continuidad necesarias para garantizar los estándares mínimos. Uno de los testimonios (cuadro 2: AL8) reflejó cómo las jerarquías de poder que se establecen entre los involucrados en el control del cáncer constituyen un obstáculo para la adecuada articulación de los componentes del programa.

Debido a los insuficientes recursos disponibles hubo demoras en la entrega de resultados, tanto de las pruebas citológicas como de las biopsias. La falta de continuidad en la asignación de los recursos se explica por la poca relevancia que se da al cáncer cervicouterino como problema de salud en los departamentos estudiados. De hecho en el nuevo laboratorio de salud pública de ese departamento no se programaron recursos para el control de la calidad de las pruebas citológicas a pesar de tener un área física prevista para ello (cuadro 3: P8).

\section{DISCUSIÓN}

Los hallazgos explican en parte el bajo impacto sobre la mortalidad que han tenido las actividades de detección temprana del cáncer cervicouterino. La infección por el VIH/sida, la salud reproductiva y otros problemas considerados explícitamente en los Objetivos de Desarrollo del Milenio (15) han encontrado una mayor respuesta de los gobiernos nacionales debido a los compromisos internacionales inherentes. Los planes regionales y municipales para el control del cáncer cervicouterino, en cambio, presentan deficiencias en su estructura y han recibido una baja asignación de recursos. Esta situación, también observada hace una década en Perú, puede estar relacionada con el cumplimiento de la recomendación hecha por la OMS de integrar el control del cáncer cervicouterino en los programas de salud sexual y reproductiva (16), donde el tamizaje se realiza con frecuencia en la población de edad fértil, particularmente la de bajo riesgo (17). En consecuencia, y a pesar de que el adecuado control del cáncer cervicouterino puede reflejar el nivel de equidad de un sistema de salud, la elevada mortalidad por esta causa no ha tenido la misma atención que se da a otros problemas de salud considerados referentes del desarrollo social $(18,19)$.

Este estudio revela un retroceso en los programas de detección temprana del cáncer cervicouterino, lo que se relaciona con las particularidades del contexto político y económico — como las dificultades en los procesos de descentralización y una exagerada orientación financiera-, así como con las características del contexto del sistema de salud, entre ellas la inadecuada planificación normativa. La reforma del sistema nacional de salud de Colombia, que llevó al establecimiento de un sistema general de seguridad social en 1993, impulsó la descentralización política y administrativa y otorgó una autonomía creciente al nivel municipal, sin una consideración especial para los programas de salud pública (20). Esto se ha traducido en menos actividades de detección temprana y marcadas diferencias entre las unidades territoriales (e incluso al interior de una misma unidad) por la fragmentación de la población según el régimen de seguro de salud o la empresa aseguradora. A esto se unen deficiencias en la asistencia técnica y el control debido a la falta de capacidad de los niveles inferiores.

Por otra parte, se comprobó que se reemplazaron los programas estructurados por normas que establecen acciones obligatorias, metas de programación y mecanismos de evaluación. Los resultados demuestran una gran heterogeneidad en la interpretación de las normas y una tendencia a limitarse a lo que está estrictamente definido en ellas. Estas consecuencias conocidas de la planificación normativa (21) se suman a la dificultad para modificar el curso de las acciones una vez en operación. Esta modalidad de planificación entró en desuso tras el auge de la planificación estratégica, pero podría resultar apropiada para el concepto de mercado regulado que rige el sistema de salud en Colombia, ya que supone que son los actores individuales (en este caso las empresas aseguradoras) los indicados para seleccionar y ejecutar los programas. De esta forma, la planificación solo racionaliza la implementación de las estrategias de desarrollo, pero no la coordina ni la controla.

El elevado interés por el flujo de recursos refleja la orientación financiera y de mercado, lo que desvirtúa el objetivo verdadero, que es controlar la enfermedad. El sistema colombiano pone un importante énfasis en la eficiencia institucional, lo que trae como consecuencia limitaciones para el acceso oportuno a los servicios -debido a los mecanismos de contención de costos- y falta decontinuidad en los procesos - por la reduc- 
ción de nóminas y la búsqueda de formas de contratación menos costosas-, aspectos ya observados al analizar el control de la tuberculosis en Colombia (22).

Otros factores relacionados con la descentralización (la alta rotación con cada cambio de administración) y la planificación normativa (la capacitación y el desarrollo de competencias técnicas como responsabilidad aislada de diferentes actores) también dificultan mantener los recursos humanos idóneos. La baja capacidad técnica y la organización y gestión subóptimas que esto implica -elementos observados también en otros países de América Latina (23) — se originan, además, en factores no contemplados en este estudio, como la deficiente formación académica.

En Colombia se ha alcanzado un nivel promedio de cobertura de las pruebas citológicas superior a $70 \%$, si bien en los departamentos estudiados esa cobertura no ha superado $65 \%$ en los últimos tres años (24). Esto se ha logrado a pesar de las dificultades ya mencionadas, como la toma de muestras a mujeres ya tamizadas, la inclusión de adolescentes, etc. La elevada cobertura puede deberse en muchos casos a la acción de organizaciones no gubernamentales y otros actores externos al sistema de salud, que en ocasiones llegan a suplir la responsabilidad del Estado (25). Es posible que la mayor parte de la mortalidad asociada se concentre en la población no asegurada, algo que queda aún por comprobar.

En términos generales, los programas implementados en América Latina, orientados por los resultados obtenidos en países desarrollados, han puesto mayor énfasis en alcanzar una elevada cobertura (26), sin embargo, se debe resaltar que en los países desarrollados se hace un seguimiento adecuado y las pruebas citológicas se realizan con buena calidad. En este estudio se comprobó que en los departamentos estudiados, el seguimiento ha presentado dificultades por la asignación de responsabilidades a múltiples actores sin una dirección central. La falta de continuidad en la confirmación diagnóstica y el tratamiento -un problema frecuente en América Latina (27, 28) — no se ha evaluado adecuadamente y podría ser uno de los factores principales que reducen el impacto. En los países latinoamericanos en los que se ha logrado una amplia cobertura de tamizaje tampoco se ha visto una reducción signi- ficativa de la mortalidad por cáncer cervicouterino $(29,30)$.

La calidad de las pruebas citológicas es un atributo al que frecuentemente se imputa la falta de impacto de los programas de tamizaje. Hay pocas publicaciones que evalúan la calidad de estas pruebas en los países latinoamericanos y las existentes muestran resultados contradictorios que, con pocas excepciones, han mejorado con el paso de los años (31-33). A pesar de que los estándares internacionales recomiendan tener laboratorios centralizados de lectura de las pruebas citológicas para mejorar la calidad del diagnóstico, en los últimos años en Colombia ha aumentado el número de laboratorios que analizan las pruebas citológicas como resultado de la competencia regulada y la descentralización. Esto afecta de forma negativa a la calidad final del diagnóstico. A ello se suma la ausencia de dirección central y la falta de acciones para el control de la calidad, como se observó en el presente estudio; no obstante, la información obtenida no permite hacer una valoración objetiva de este aspecto.

México y Chile son, quizá, los dos únicos países de América Latina que han logrado reducir la mortalidad por cáncer cervicouterino. Las evaluaciones del programa mexicano orientaron su reorganización con el objetivo de aumentar la cobertura en grupos de mujeres de alto riesgo, regular la práctica de los colposcopistas y técnicos de citología, establecer sistemas de vigilancia epidemiológica y crear mecanismos de aseguramiento de la calidad del diagnóstico, el seguimiento y el tratamiento de los casos (34). En Chile, la reducción de la mortalidad se atribuye al aumento de la cobertura y la implementación del control de la calidad en los laboratorios - tanto interno como externo-, pero se debe resaltar que la atención oportuna de las pacientes con lesiones cervicouterinas mejoró considerablemente en ese país después de la promulgación de la norma sobre la oportunidad del diagnóstico y el inicio del tratamiento (35). En estos países se cuenta con programas bien organizados, lo que respalda lo anotado por diversos autores acerca de la falta de efectividad de los sistemas de tamizaje que no buscan activamente a las mujeres en riesgo, que no cuentan con sistemas validados de control de la calidad y que no contemplan el adecuado seguimiento de las mujeres con resultados alterados en sus pruebas citológicas $(21,36)$.

Una importante limitación del presente trabajo es que no se tuvieron en cuenta las opiniones de las mujeres - que como sujetos de investigación hubieran aportado elementos enriquecedores- ni de las autoridades del Ministerio de Salud, que con seguridad deben tener una perspectiva diferente de la situación. Estas limitaciones deben tomarse en cuenta en el momento de valorar los resultados expuestos. Por otra parte, los actores incluidos en el estudio no identificaron aspectos positivos en el estado actual de las acciones de detección temprana, lo cual podría interpretarse como un sesgo en el análisis de los datos. No obstante, las convocatorias realizadas fueron atendidas ampliamente y los procedimientos del estudio aseguraron la inclusión de distintas perspectivas del sistema de salud (representantes de las autoridades de gobierno, las empresas aseguradoras, los servicios de salud y los profesionales, entre otros).

Como conclusión se puede afirmar que hay tres elementos del contexto que hacen difícil la articulación de las diferentes actividades para el control del cáncer cervicouterino en Colombia: la orientación financiera, la planificación normativa y las dificultades de la descentralización. Estos elementos impiden que las entidades puedan cumplir adecuadamente con sus diferentes objetivos propuestos para el control del cáncer cervicouterino, en particular la cobertura de la población de alto riesgo, el seguimiento de las mujeres con resultados alterados en sus pruebas citológicas, el control de la calidad de las pruebas y, especialmente, el acceso al diagnóstico definitivo y al tratamiento oportuno.

En los países donde las acciones de tamizaje están organizadas de forma fragmentada, como en Colombia, las diversas instituciones deben colaborar en el diseño e implementación de programas integrados de tamizaje. Esta recomendación es válida incluso si el tamizaje se basa en pruebas citológicas diferentes, incluida la detección de ADN del virus del papiloma humano. Es necesario articular los diferentes componentes y actores involucrados en el control del cáncer cervicouterino mediante una dirección central y una acción descentralizada, según las particularidades de cada sistema de salud. Por último, se recomienda promover la participación de los 
diferentes actores del sistema de salud para llegar a una planificación prospectiva centrada en los objetivos de la salud pública mas que en la contención de los costos y las metas programáticas asociadas con el cumplimiento de las normas.
Agradecimientos. A todos los funcionarios de las direcciones departamentales, municipales e instituciones de salud que participaron en las entrevistas y grupos focales, en particular a Zandra Lucía Castañeda, Ruth Jael Robles Santos,
Melva Ortiz, Carlos Ricardo Escobar y Edgar Salguero, por su apoyo operativo para el desarrollo del proyecto. Esta investigación fue realizada con recursos de inversión del Instituto Nacional de Cancerología, Bogotá, Colombia.

\section{REFERENCIAS}

1. Canfell K, Sitas F, Beral V. Cervical cancer in Australia and the United Kingdom: comparison of screening policy and uptake, and cancer incidence and mortality. Med J Aust. 2006; 185(9):482-6.

2. Kitchener $H$, Castle P, Cox J. Achievements and limitations of cervical cytology screening. Vaccine. 2006;24(supl 3):63-70.

3. Ferlay J, Bray F, Pisani P, Parkin DM. Cancer incidence, mortality, and prevalence worldwide. Lyon: IARC Press; 2002.

4. Arrossi S, Sankaranarayanan R, Parkin DM. Incidence and mortality of cervical cancer in Latin America. Salud Publica Mex. 2003;45 (supl 3):S306-14.

5. Sankaranarayanan R, Madhukar-Budukh A, Rajkumar R. Effective screening programmes for cervical cancer in low- and middle-income developing countries. Bull World Health Organ. 2001;79(10):954-62.

6. Globocan 2002 [programa informático]. Lyon: International Agency for Research on Cancer; 2004.

7. Homedes N, Ugalde A. Neoliberal reforms in health services in Latin America: a critical view from two case studies. Rev Panam Salud Publica. 2005;17(3):210-20.

8. Hernández M. Reforma sanitaria, equidad y derecho a la salud en Colombia. Cad Saude Pública. 2002;18(4):991-1001.

9. Almeida C. Reforma de sistemas de servicios de salud y equidad en América Latina y el Caribe: algunas lecciones de los años 80 y 90 . Cad Saude Publica. 2002;18(4):905-25.

10. República de Colombia, Ministerio de Salud. Resolución 412. Santa Fe de Bogotá: Ministerio de Salud de Colombia; 2000.

11. Fundación Corona. Proyecto Así Vamos en Salud: aseguramiento en salud 2000-2006 [sitio en Internet]. Santa Fe de Bogotá: Fundación Santa Fe de Bogotá; 2006. Disponible en http:/ / www.asivamosensalud.org/areas / aseguramiento.htm. Acceso el 29 de julio de 2008.

12. Murillo R, Piñeros M, Hernández G. Atlas de mortalidad en Colombia. Santa Fe de Bogotá: Instituto Nacional de Cancerología, Instituto Geográfico Agustín Codazzi; 2003.

13. Glaser B, Strauss A. The discovery of grounded theory. Strategies for qualitative research. New York: Aldine; 1967.
14. Strauss A, Corbin J. Basics of qualitative research. Grounded theory procedures and techniques. London: Sage Publications; 1990.

15. Naciones Unidas. Objetivos de Desarrollo del Milenio: una mirada desde América Latina y el Caribe. Santiago: ONU; 2005.

16. Pan American Health Organization. Program for the control of cervical cancer in Peru. Bull Pan Am Health Organ. 1996;30(4):413.

17. Eluf-Neto J, Nascimento CM. Cervical cancer in Latin America. Semin Oncol. 2001;28(2): 188-97.

18. Lorant V, Boland $B$, Humblet $P$, Deliège $D$. Equity in prevention and health care. J Epidemiol Community Health. 2002;56:510-6.

19. Baker D, Middleton E. Cervical screening and health inequality in England in the 1990s. J Epidemiol Community Health. 2003;57(6): 417-23.

20. Ugalde A, Homedes N. Descentralización del sector salud en América Latina. Gac Sanit. 2002;16(1):18-29.

21. Barrenechea J, Trujillo E, Chorny A. Salud para todos en el año 2000. Implicaciones para la planificación y administración de los sistemas de salud. Medellín: Editorial Universidad de Antioquia; 1990.

22. Arbeláez MP, Gaviria MB, Franco A, Restrepo R, Hincapié D, Blas E. Tuberculosis control and managed competition in Colombia. Int J Health Plann Manage. 2004;19(supl1):S25-43.

23. Lewis MJ. Análisis de la situación del cáncer cervicouterino en América Latina y el Caribe. Washington, D.C.: Organización Panamericana de la Salud; 2004.

24. Piñeros M, Cendales R, Murillo R, Wiesner C, Tovar S. Cobertura de la citología de cuello uterino y factores relacionados en Colombia, 2005. Rev Salud Publica. 2007;9(3):327-41.

25. Wiesner-Ceballos C, Vejarano-Velandia M, Caicedo-Mera JC, Tovar-Murillo SL, CendalesDuarte R. La citología de cuello uterino en Soacha, Colombia: representaciones sociales, barreras y motivaciones. Rev Salud Publica. 2006;8(3):185-96

26. Quinn M, Babb P, Jones J, Allen E. Effect of screening on incidence of and mortality from cancer of cervix in England: evaluation based on routinely collected statistics. Br Med J. 1999;318(7188):904-8.

27. Lazcano-Ponce EC, Nájera-Aguilar P, Buiatti E, Alonso-de-Ruiz P, Kuri P, Cantoral L, et al.
The cervical cancer screening program in Mexico: problems with access and coverage. Cancer Causes Control. 1997;8(5):698-704.

28. Donoso E, Cuello M, Villarroel L. Reducción de la mortalidad por cáncer cérvico uterino en Chile, 1990-2003. Rev Chil Obstet Ginecol. 2006;71(5):307-12.

29. Pan American Health Organziation. Evaluation of the cervical cancer control program in Cuba. Bull Pan Am Health Organ. 1996;30(4): 387-91.

30. De Araujo-Pinho A, França I, Schraiber LB, D'Oliveira AF. Cervical cancer screening in the municipality of São Paulo: coverage and factors involved in submitting to the Pap test. Cad Saude Publica. 2003;19(supl2):S303-13.

31. Howe SL, Vargas DE, Granada D, Smith JK. Cervical cancer prevention in remote rural Nicaragua: a program evaluation. Gynecol Oncol. 2005;99(3 supl1):S232-5.

32. Parentoni-Costa CR, Ávila-Fernandes P. National cervical cancer screening program: the contribution of anatomic pathology laboratory of Santa Casa de Misericordia de Passos (MG). Rev Bras Cancerol. 2003;49(1):33-7.

33. Capurro I, Rojo JA, Pino T, Velásquez C, Garay J, Venegas M. Programa de detección y control de cáncer de cuello uterino en servicio salud Araucania Sur. Rev Chil Obstet Ginecol. 2006;71(5):307-12.

34. Lazcano-Ponce E, Nájera-Aguilar P, Alonsode-Ruiz P, Buiatti E, Hernández-Ávila M. Programa de detección oportuna de cáncer cervical en México. Propuesta de reorganización. Rev Inst Nac Cancerol (Mex). 1996; 42(3):141-58.

35. República de Chile, Ministerio de Salud. Ley No. 19.966, establece las garantías en salud. Santiago: Ministerio de Salud; 2004.

36. Centers for Disease Control and Prevention. Strategies for providing follow-up and treatment services in the National Breast and Cervical Cancer Early Detection ProgramUnited States, 1997. J Am Med Assoc. 1998; 279(24):1941-2

Manuscrito recibido el 3 de septiembre de 2007. Aceptado para publicación, tras revisión, el 27 de mayo de 2008. 
ABSTRACT Objectives. To characterize the health system stakeholder's perspective on the basics of the political, economic, and sanitary context, as well as the ways in which control activities are being realized in four of Colombia's health departments.

Control of cervical cancer in Colombia: the perspective of the health system

Key words Uterine cervical neoplasms, prevention and control; health services; qualitative research; Colombia. 